\title{
Numerical and experimental analysis of sensitivity enhanced RI sensor based on Ex-TFG in thin cladding fiber
}

\author{
Zhijun Yan ${ }^{1,2}$, Zhongyuan Sun ${ }^{1}$,Kaiming Zhou ${ }^{1, *}$, Binbin Luo ${ }^{1,3}$, Jianfeng Li ${ }^{1,4}$, Hushan Wang $^{2}$, \\ Yishan Wang ${ }^{2}$, Wei, Zhao ${ }^{2}$, Lin Zhang ${ }^{1}$
}

\begin{abstract}
We report a highly sensitive refractive index (RI) sensor in the aqueous solution, which is based on an $81^{\circ}$-tilted fiber grating $\left(81^{\circ}\right.$-TFG) structure inscribed into a thin cladding fiber with $40 \mu \mathrm{m}$ cladding radius. The numerical analysis has indicated that the RI sensitivity of cladding resonance mode of the grating can be significantly enhanced with reducing cladding size. This has been proved by the experimental results as the RI sensitivities of TM and TE resonance peaks in the index region of 1.345 have been increased to $1180 \mathrm{~nm} / \mathrm{RIU}$ and $1150 \mathrm{~nm} / \mathrm{RIU}$, respectively, compared to only $200 \mathrm{~nm} / \mathrm{RIU}$ and $170 \mathrm{~nm} / \mathrm{RIU}$ for the same grating structure inscribed in standard telecom fiber with $62.5 \mu \mathrm{m}$ cladding radius. Although the temperature sensitivity has also increased, the change in temperature sensitivity is still insignificant in comparison with RI sensitivity enhancement.
\end{abstract}

Index Terms-Tilted fiber grating, Refractive index sensing, Sensitivity.

\section{INTRODUCTION}

O ptical fiber grating based sensors have been extensively studied and developed for real applications in past decades. Recently, refractive index (RI) sensors based on fiber grating have attracted more interests, providing a new type of chemical and biological sensor platform with high sensitivity and selectivity. The principle is based on the fact that RI of surrounding medium can affect the mode coupling induced by the grating, which can be embodied by resonant peaks. Not all fiber gratings are suitable for RI sensing; only those having evanescence field penetrating to the surrounding medium. This can only be facilitated by the grating structure which is able to couple the light from core mode to cladding modes[1, 2]. The theoretical analysis shows that the coupled forward-propagating cladding modes have almost 1000 times higher sensitivity than the backward-propagating ones [3]. There are only two types of grating that can couple the core mode to forward-propagating cladding modes: long period grating (LPG) and excessively tilted fiber grating (Ex-TFG). The LPGs have been extensively developed and investigated in

Manuscript submitted XXXXX

${ }^{1}$ Aston Institute of Photonic Technologies, Aston University, Birmingham, UK, B4 7ET

${ }^{2}$ State Key Laboratory of Transient Optics and Photonics, Xi'an Institute of Optics and Precision Mechanics, Chinese Academy of Sciences, Xi'an 710119, China

${ }^{3}$ Department of Electronic Engineering, Chongqing University of Technology, Chongqing, 400050, China

${ }^{4}$ School of Optoelectronic Information, University of Electronic Science and Technology of China, Chengdu 610054, China

Corresponding email: k.zhou@aston.ac.uk last two decades, mainly as attenuation filters and environment detectors [4-11]. In contrast, Ex-TFGs are relatively new and have not been explored extensively. Firstly reported by Zhou, the Ex-TFGs have dual-peak resonances in spectrum corresponding to two sets of polarization dependent modes caused by the asymmetric structure induced by the excessively tilted index fringes[12, 13]. Comparing with normal LPG, the Ex-TFG shows much higher sensitivity in the lower RI region, but it is still not high enough to detect small changes in the aqueous solution with RI around 1.33-1.35. To this end, the cladding mode of much higher order (>40th) should be used. However, from the fabrication point of view, it is difficult to fabricate high quality gratings that can achieve strong coupling between core mode and the high order cladding modes, as their coupling coefficients are intrinsically low [14]. Recently, much more efforts have been made to improve the sensitivity for aqueous solutions, such as: (a) coating a high RI layer on the fiber cladding, thus moving the resonance cladding modes to the dispersion turning point[15-17] and (b) reducing cladding layer thickness to enhance the dependence of the effective index of the cladding modes on the ambient refractive index [7, 18, 19]. Although by designing the LPG at turning point or coating high refractive index film outside the cladding, the RI sensitivity of LPG can be greatly improved in the aqueous, the spectral of resonance peaks have very low $\mathrm{Q}$ factor, which is not desirable for real applications [7, 20]. In contrast, Ex-TFGs are capable of light coupling to much higher order cladding modes, shifting the sensitive region to lower RI end, and has very high $\mathrm{Q}$ factor.

In this paper, we will report the theoretical analysis and experimental investigation of thermal and RI sensitivity of Ex-TFGs which have been UV-inscribed in the fiber with small cladding diameter.

\section{THEORETICAL ANALYSIS}

Due to the highly asymmetrical index fringe structure in the fiber core (image shown in Figure 4)an Ex-TFG induces two sets of birefringence cladding modes, thus causing pronounced polarization dependent loss associated with TM/TE resonance peaks. The mode coupling of fiber grating is determined by the phase matching condition. For Ex-TFGs, the strongest coupling between the core and co-propagating cladding modes can be expressed by following phase matching condition [13]:

$$
\begin{gathered}
\lambda=\left(n_{c o}^{\text {eff }}(\lambda, T, \varepsilon)-n_{c l, m}^{i, e f f}\left(\lambda, n_{S R I}, T, \varepsilon\right)\right) \frac{\Lambda_{G}(T, \varepsilon)}{\cos \theta} \\
\mathrm{i}=\mathrm{TE} \text { or TM }
\end{gathered}
$$


Where $\lambda$ is the resonance wavelength, $n_{c o}^{\text {eff }}$ is the effective index of the core mode state, $\varepsilon$ and $\mathrm{T}$ are the strain and temperature applied to the fiber, respectively, $n_{c l, m}^{i, e f f}$ is the effective index of $m^{\text {th }}$ cladding mode at the wavelength $\lambda, n_{S R I}$ is the refractive index of surrounding medium ${ }_{*} \Lambda_{G}$ is the normal period of grating and $\theta$ is the tilt angle of the grating.

From Equation 1, we can find that the sensing nature of grating is the effective indexes of the cladding modes are changing with the environmental condition. Particularly, the effective index of cladding mode is affected by the interaction between the surrounding medium and the evanescence field of guided mode. However, the depth of evanescence field of core mode is only several micrometers and does not spread to outside of the fiber cladding, thus would be insensitive to the index change of surrounding medium. By using the analysis method reported in [21], we can obtain the general sensitivity expression of cladding mode:

$$
\begin{aligned}
\Delta \lambda & =\frac{1}{1-\left(\frac{d n_{c o}^{e f f}}{d \lambda}-\frac{d n_{c l, m}^{e f f}}{d \lambda}\right) \Lambda}\left(\frac{\left(\delta n_{c o}^{e f f}-\delta n_{c l, m}^{e f f}\right)}{n_{c o}^{e f f}(\lambda)-n_{c l, m}^{e f f}(\lambda)}+\frac{d \Lambda}{\Lambda}\right) \lambda \\
& =\gamma(\Gamma+\alpha) \lambda
\end{aligned}
$$

Where, $\gamma$ is the waveguide dispersion, second term is the dependence of waveguide dispersion $(\Gamma)$ on environment and the material expansion $(\alpha)$ caused by the changing of environment, and the third one is the operation wavelength. In Equation 2, the RI and temperature dependence of waveguide dispersion $\left(\Gamma_{\mathrm{RI}}\right.$, and $\left.\Gamma_{\mathrm{TEM}}\right)$ are expressed as [5]:

$$
\Gamma_{R I}=-\frac{u_{m}{ }^{2} \lambda^{2} \Lambda n_{\text {sur }}}{8 \pi^{3} r^{3} n_{c l, m}^{e f f}\left(n_{c l}{ }^{2}-n_{\text {sur }}{ }^{2}\right)^{3 / 2}}
$$

Where, $u_{m}$ is $m^{\text {th }}$ root of the zero order Bessel function $\left(J_{0}(u)=0\right) ; r$ is the radius of cladding; $n_{s u r}$ is the RI of surrounding medium; $n_{c l}$ is the index of cladding; $n_{c l}{ }^{e f f}$ is the effective index of cladding mode; $\lambda$ is the operation wavelength of cladding mode[5].

$$
\Gamma_{T E M}=\frac{\left(\xi_{c o} n_{c o}^{e f f}-\xi_{c l} n_{c l, m}^{e f f}\right)}{n_{c o}^{e f f}(\lambda)-n_{c l, m}^{e f f}(\lambda)}
$$

Where, $\xi_{c o}$ and $\xi_{c l}$ are the thermo-optic coefficients of core and cladding materials.

As we have mentioned each corresponding mode of an Ex-TFG will split into two orthogonal polarization (TE and TM) resonance peaks, and the TE and TM resonance peaks have the same sensitivity variation tendency when the environment condition has been changed. So, in our simulation, we only analyze the TM peak with different cladding sizes.
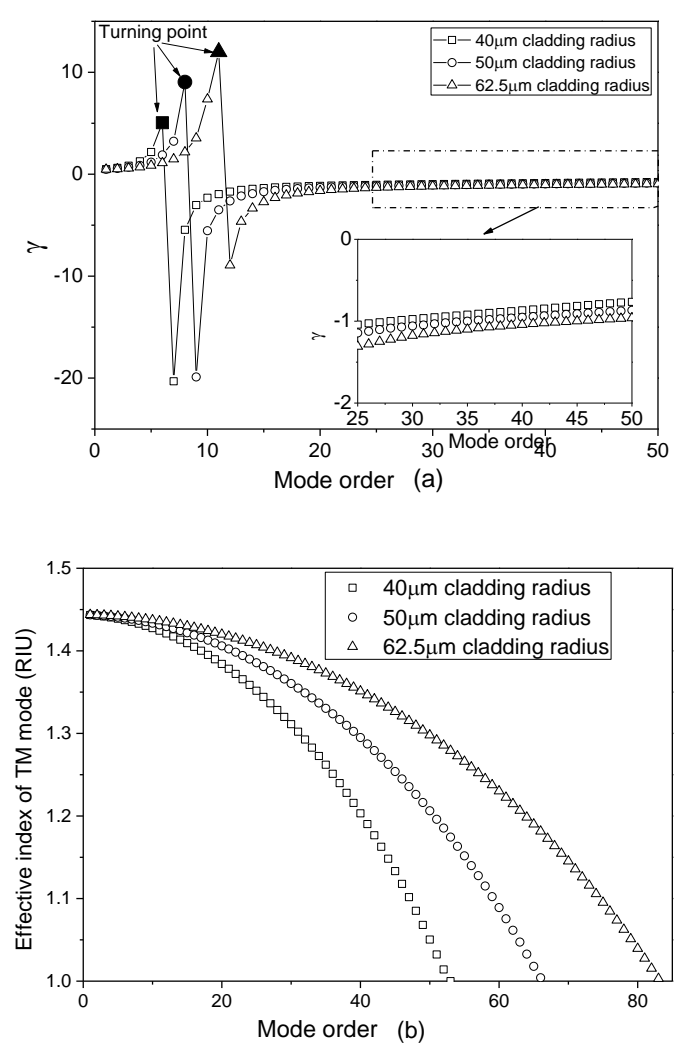

Figure 1 (a) $\gamma$ factor and (b) effective index of cladding mode with TM polarization at $1550 \mathrm{~nm}$ for fibers with different cladding radii: $40 \mu \mathrm{m}$ (open square), $50 \mu \mathrm{m}$ (open circle) and $62.5 \mu \mathrm{m}$ (open triangle).

It has been indicated in Equation 2 that the environmental sensitivity depends on $\gamma, \Gamma, \alpha$ and $\lambda$. As we can clearly see from Equation 3, $\Gamma_{R I}$ is inversely proportion to the $r^{3}$ and $n_{\text {eff. }}$ So, by reducing the fiber cladding size, the $\Gamma_{\mathrm{RI}}$, thus the RI sensitivity, can be greatly increased. In our simulation, we compared three different cladding size fibers $(40 \mu \mathrm{m}, 50 \mu \mathrm{m}$ and $62.5 \mu \mathrm{m})$. The $\gamma$ factor of cladding mode with TM polarization at $1550 \mathrm{~nm}$ is plotted in Figure 1(a). As it shown, the dispersion turning point moves to the low order cladding mode when the fiber cladding radius reduced. At the turning point, the $\gamma$ factor changes from positive to negative. Because of very short period we used which is $6.6 \mu \mathrm{m}$, the responding cladding mode order of Ex-TFGs fabricated for this investigation is higher than $20^{\text {th }}$. The inset of Figure 1(a) shows enlarged figure of $\gamma$ factor at mode order $>20^{\text {th }}$, in which the $\gamma$ factor does not change too much. Figure 1 (b) shows the simulated effective indexes of cladding mode with TM polarization at $1550 \mathrm{~nm}$ for fibers with different cladding radii. Because the number of guided cladding modes is determined by the radius of cladding, we see from the figure that the number of guided modes is decreasing as the cladding radius reducing. For the same order modes, the smaller cladding radius is, the lower the cladding mode effective index. According to the calculation, the effective index of the $25^{\text {th }}$ cladding mode with TM polarization is 1.407 , 1.390 and 1.352 for the fibers with a cladding radius of $62.5 \mu \mathrm{m}$, $50 \mu \mathrm{m}$ and $40 \mu \mathrm{m}$, respectively. 

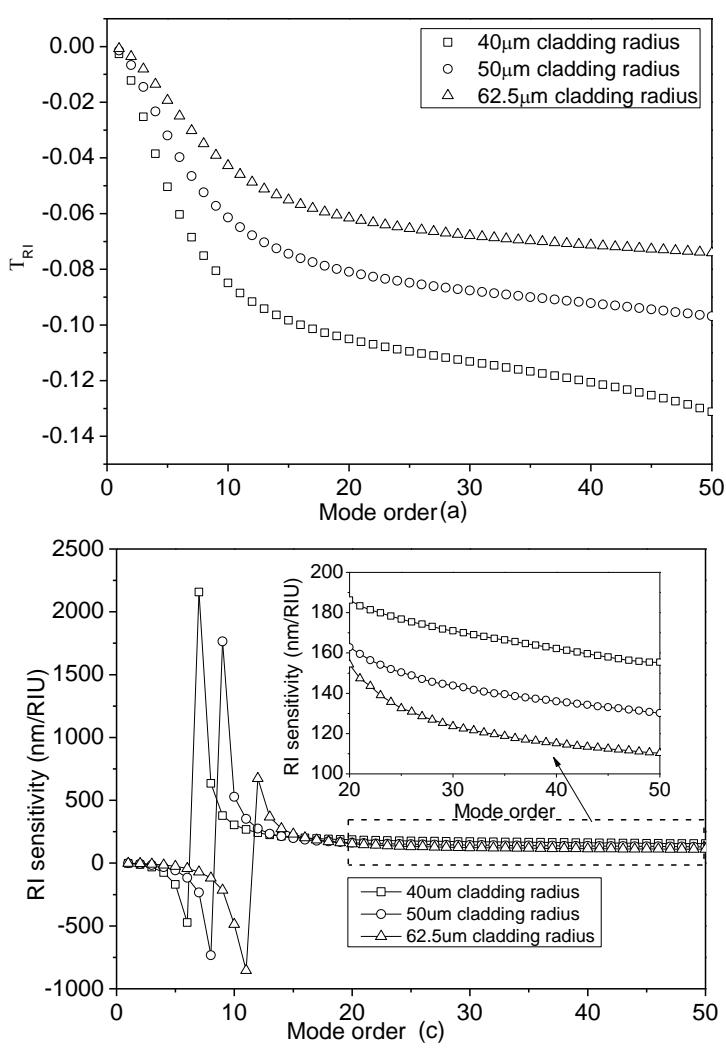

Figure 2 (a) RI dependency on waveguide dispersion $\boldsymbol{\Gamma}_{\boldsymbol{R} I}$ and (b) RI sensitivity (inset: enlarged view for dashed part) of cladding mode with TM polarization at $1550 \mathrm{~nm}$ for fibers with different cladding radii: $40 \mu \mathrm{m}$ (open square), $50 \mu \mathrm{m}$ (open circle) and $62.5 \mu \mathrm{m}$ (open triangle).

Figure 2 (a) and (b) show the calculated RI dependency on waveguide dispersion and sensitivity for fibers with different cladding radii at the surrounding medium index 1.345. As it shown in Figure 2 (a), the $\Gamma_{R I}$ for the $40 \mu \mathrm{m}$ cladding radius is almost twice of the fiber of $62.5 \mu \mathrm{m}$ cladding radius. Also, the RI sensitivity is increasing when the cladding radius is reduced from $62.5 \mu \mathrm{m}$ to $40 \mu \mathrm{m}$ for the high order cladding mode (see in Figure 2 (b)). The simulated results show that the RI sensitivity can be significantly increased by using the small cladding radius fiber, which has been verified in the experiment described below.

According to Equation 4, we can find that for $\xi_{c o}>\xi_{c l}, \Gamma_{T E M}$ is always a positive value and approaching to zero with increasing mode order. While for $\xi_{c o}<\xi_{c l}$, there is a switchover point at which $\Gamma_{T E M}$ is equal to zero and after which it changes from negative to positive. For pure silica, $\xi_{c l}$ is $7.8 \times 10^{-6} /{ }^{0} \mathrm{C}[22]$, and $\xi_{c o}$ depends on the dopants and their concentrations in the fiber core [23]. In the experiment, the fibers we used are SMF-28 (from Corning) with core to cladding ratio as 9/125 and SM1500 (from FiberCore) as 4.2/80. The $\xi_{c o}$ value can be obtained by measuring the temperature sensitivity of a fiber Bragg grating (FBG) inscribed into the fiber. Then, we obtained the temperature sensitivities of the FBGs UV inscribed in SMF-28and SM1500 fibers at around $11.6 \mathrm{pm} /{ }^{\circ} \mathrm{C}$ and $8.26 \mathrm{pm} /{ }^{\circ} \mathrm{C}$, respectively. So, according to the calculation method we reported in Ref. [24], the $\xi_{c o}$ values of SMF-28and SM1500 fibers are $7.07 \times 10^{-6} /{ }^{\circ} \mathrm{C}$ and $8.26 \times 10^{-6} /{ }^{0} \mathrm{C}$, respectively. The thermal expansion of silica material is around $5 \times 10^{-7}$. According to the calculation from Equation 2 shows, the $35^{\text {th }}$ cladding with TM peak in SMF-28fiber has a positive thermal sensitivity, whereas the $24^{\text {th }}$ mode in SM1500 has a negative thermal sensitivity as shown in Figure 3 (b).

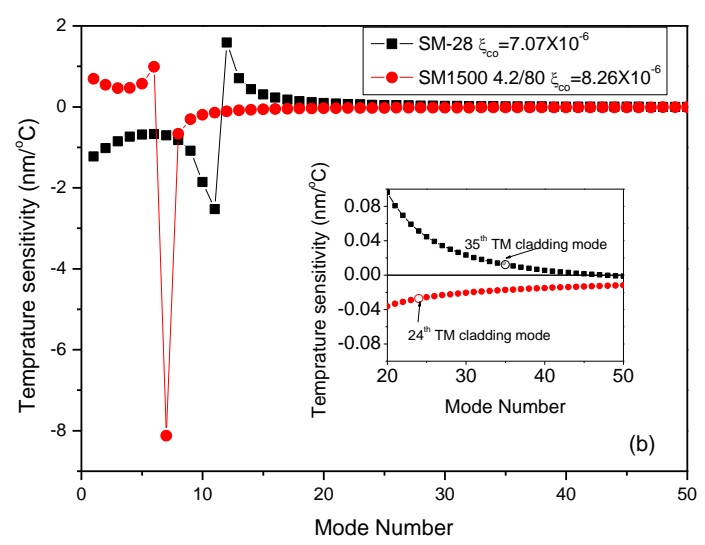

Figure 3 (a) Temperature dependency on waveguide dispersion $\boldsymbol{\Gamma}_{\boldsymbol{T E M}}$ and (b) Temperature sensitivity (inset: enlarged view for dashed part) of cladding mode with TM peaks at $1550 \mathrm{~nm}$ for SMF-28(Solid square) and SM1500 $(4.2 / 80)$ (Solid circle)

\section{FABRICATION AND EXPERIMENTAL RESULTS}

As discussed in previous section, the RI sensitivity of an Ex-TFG could be improved by using small cladding radius fiber. To verify this, we inscribed $81^{\circ}$-TFGs into SMF-28 fiber with $62.5 \mu \mathrm{m}$ cladding radius and SM1500 fiber with $40 \mu \mathrm{m}$ cladding radius, respectively, and investigated their thermal and RI sensitivities. The detailed fabrication method has been reported in Ref. [13].

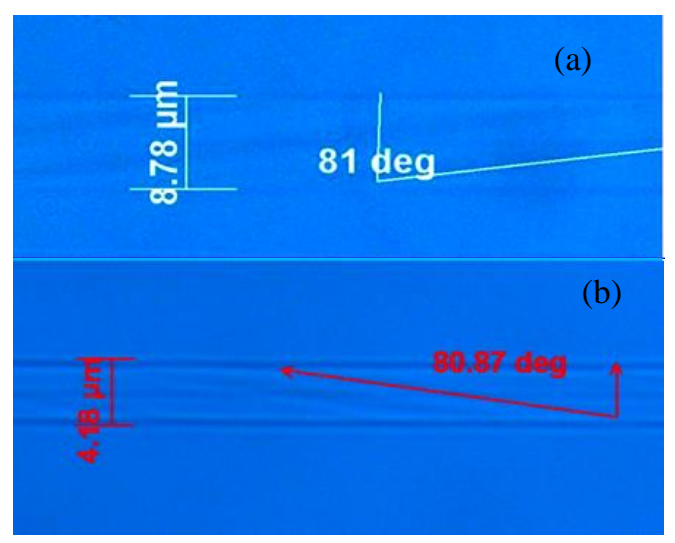




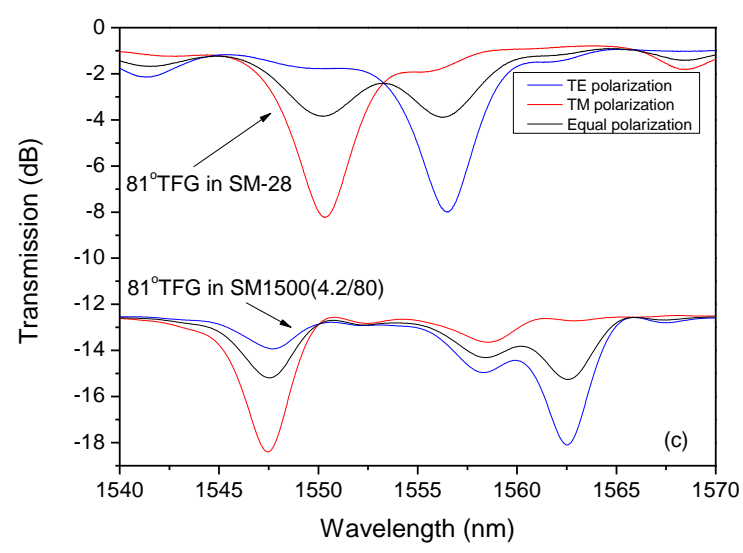

Figure 4 The micro-images of $81^{\circ}$-TFGs in (a) SMF-28and (b) SM1500 fiber; (c) their transmission spectra.

Figure 4 (a) and (b) show the micro-images of $81^{\circ}$-TFGs in SMF-28and SM1500 fibers, in which the grating pattern and fiber core size are clearly distinguished. The transmission spectra of $81^{\circ}$-TFGs in SMF-28 and SM1500 fiber are plotted in Figure 4 (c). As we can see in the figure, there are two polarization dependence resonance peaks, which are related to TM and TE cladding mode and the wavelength separations are around $7 \mathrm{~nm}$ and $14 \mathrm{~nm}$ for $81^{\circ}$-TFGs in SMF-28 and SM1500 fibers, respectively. According to the calculation, the dual-peak positions of the 35th cladding mode of $81^{\circ}$-TFG in SMF-28 fiber are at $1550 \mathrm{~nm}$ for TM and $1556.5 \mathrm{~nm}$ for TM and the 24th cladding mode in SM1500 fiber are at $1546 \mathrm{~nm}$ for TM and $1563 \mathrm{~nm}$ for TM. This gives the peal separations of $6.5 \mathrm{~nm}$ for the former and $17 \mathrm{~nm}$ for the latter, which is in reasonable agreement with the experimentally measured dual peak separation values.

Figure 5 (a) shows that the measured thermal sensitivities are $6.9 \mathrm{pm} /{ }^{\circ} \mathrm{C}$ and $5.9 \mathrm{pm} /{ }^{\circ} \mathrm{C}$ and $-21.2 \mathrm{pm} /{ }^{\circ} \mathrm{C}$ and $-20.2 \mathrm{pm} /{ }^{\circ} \mathrm{C}$ for TM and TE peaks of $81^{\circ}$-TFGs made in SMF-28 and SM1500 fibers, respectively. The negative thermal sensitivity indicates the wavelength shift is decreasing with temperature. These results are also in good agreement with our simulation results shown in Figure 3 (b). As the theoretical analysis indicated, the temperature sensitivity is mainly affected by the different fiber core dopants. Thus, fiber may be designed using different dopants or dopant concentrations to reduce temperature sensitivity.

To evaluate RI sensing capability of the Ex-TFGs, we have applied a series of index oil (from Cargille laboratory) with different index values to the gratings. The gratings were straightly clamped on to two stages at the same height without bending and axial strain. After each index oil measurement, the grating was rinsed with methanol to remove the residual oil till the original spectrum in air was restored on the optical spectrum analyzer. Figure 5 (b) shows the RI sensitivity of TM and TE peaks of $81^{\circ}$-TFGs in SMF-28 and SM1500 fibers. In the figure, we can find the RI sensitivity of TM peak is slightly higher than that of TE peak. For the $81^{\circ}$-TFG in small cladding size fiber, the RI sensitivities of TM and TE peaks are $1180 \mathrm{~nm} / \mathrm{RIU}$ and 1150/RIU, respectively, whereas for the $81^{\circ}-$ TFG in SMF-28, they are $200 \mathrm{~nm} / \mathrm{RIU}$ and $170 \mathrm{~nm} / \mathrm{RIU}$. It has clearly indicated that the RI sensitivity may increase 5 times when the cladding radius reduced by $1 / 3$. For an optical spectrum analyzer with $0.06 \mathrm{~nm}$ resolution, the theoretical minimum index change that can be detected may be as low as 5 $\times 10^{-5}$ using the Ex-TFG made in SM1500 fiber.
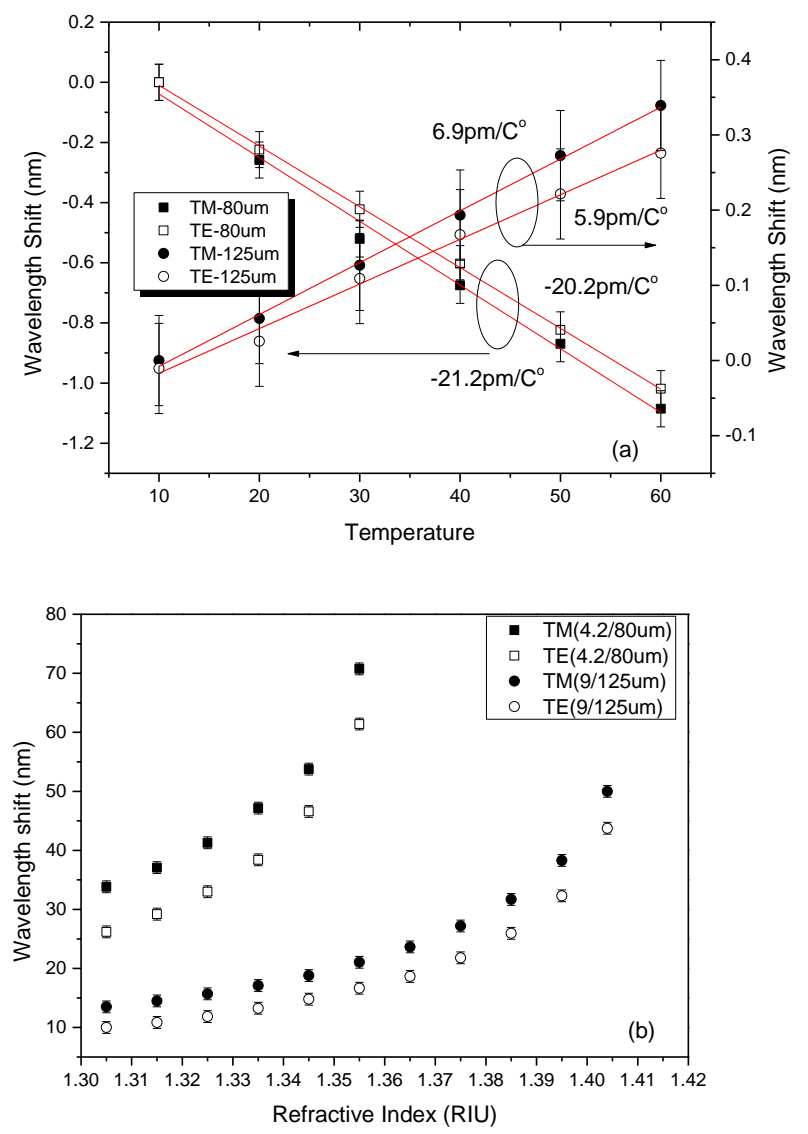

Figure 5 Comparison of (a) temperature and (b) RI response of TM (solid) and TE (open) peaks of $81^{\circ}$-TFGs inscribed into SMF-28fiber with $62.5 \mu \mathrm{m}$ cladding radius (circle) and SM1500 fiber with $40 \mu \mathrm{m}$ cladding radius (square).

\section{CONCLUSION}

We have discussed numerically and experimentally the effect of cladding radius on thermal and RI sensitivity of Ex-TFGs. The simulated results have shown that the smaller cladding radius could greatly enhance RI sensitivity, which has been also verified in the experiment. Our experimental results state that the RI sensitivity of TM and TE peak of Ex-TFG can be increased by $\sim 5$ times $(1180 \mathrm{~nm} / \mathrm{RIU}$ at the index of 1.345) for the fiber with $40 \mu \mathrm{m}$ radius cladding, compared to the fiber with $62.5 \mu \mathrm{m}$ cladding $(200 \mathrm{~nm} / \mathrm{RIU})$. For Ex-TFGs inscribed in small cladding fiber, the temperature sensitivity has been increased by $\sim 3$ times from $5.9 \mathrm{pm} /{ }^{\circ} \mathrm{C}$ to $20.2 \mathrm{pm} /{ }^{\circ} \mathrm{C}$ for the same TE peak. However, the increasing of temperature sensitivity is not due to the decreasing of core size. In the simulation analysis, we have found that the temperature 
dependency of waveguide dispersion is directly determined by the material of fiber core. By choosing suitable fiber core material, the temperature sensitivity may be reduced to eliminate thermal cross-sensitivity effect. The high RI sensitivity in aqueous solution makes Ex-TFGs UV-inscribed in small cladding fiber as suitable sensors to detect small variations in bio/chemical medium.

Acknowledgements

The research leading to these results has received funding from the European Union Seventh Framework Programme [FP7/2007-2013] under grant agreement No [295208].

\section{REFERENCES}

1. Fan, X.W., I. M.; Shopova, S. I.; Zhu, H.; Suter, J. D.; Sun, Y. , Sensitive optical biosensors for unlabeled targets: A review. Anal. Chim. Acta 2008. 620: p. 19.

2. Baldini, F., et al., Optical fibre gratings as tools for chemical and biochemical sensing. Analytical and Bioanalytical Chemistry, 2012. 402(1): p. 109-116.

3. Galina Nemova , R.K., Theoretical model of a planar integrated refractive index sensor based on surface plasmon-polariton excitation. Optics Communications, 2007. 275: p. p76-82.

4. Vengsarkar, A.M., et al., Long-period fiber gratings as band-rejection filters. Lightwave Technology, Journal of, 1996. 14(1): p. 58-65.

5. Shu, X., L. Zhang, and I. Bennion, Sensitivity Characteristics of Long-Period Fiber Gratings. J. Lightwave Technol., 2002. 20(2): p. 255.

6. Bhatia, V., Applications of long-period gratings to single andmulti-parameter sensing. Opt. Express, 1999. 4(11): p. 457-466.

7. Pilla, P., et al., Giant sensitivity of long period gratings in transition mode near the dispersion turning point: an integrated design approach. Opt. Lett., 2012. 37(19): p. 4152-4154.

8. Pilla, P., et al., Transition mode long period grating biosensor with functional multilayer coatings. Opt. Express, 2011. 19(2): p. 512-526.

9. Patrick, H.J., A.D. Kersey, and F. Bucholtz, Analysis of the response of long period fiber gratings to external index of refraction. Lightwave Technology, Journal of, 1998. 16(9): p. 1606-1612.

10. Xuewen, S., et al., High sensitivity of dual resonant peaks of long-period fibre grating to surrounding refractive index changes. Electronics Letters, 1999. 35(18): p. 1580-1581.

11. Chen, X., et al., Dual-peak long-period fiber gratings with enhanced refractive index sensitivity by finely tailored mode dispersion that uses the light cladding etching technique. Appl. Opt., 2007. 46(4): p. 451-455.

12. Zhou, K., et al., Optic sensors of high refractive-index responsivity and low thermal cross sensitivity that use fiber Bragg gratings of $80^{\circ}$ tilted structures. Opt. Lett., 2006. 31(9): p. 1193-1195.

13. Kaiming Zhou, L.Z., Xianfeng Chen, and Ian Bennion, Low Thermal Sensitivity Grating Devices Based on
Ex-45 Tilting Structure Capable of Forward-Propagating Cladding Modes Coupling. Journal of lightwave technology, 2006. 24(16): p. 5087-5094.

14. Erdogan, T., Cladding-mode resonances in short- and long-period fibre grating filters. J. Opt. Soc. Am. A, 1997. 14: p. 1760-1773.

15. Rees, N.D., et al., Optical fiber long-period gratings with Langmuir?Blodgett thin-film overlays. Opt. Lett., 2002. 27(9): p. 686-688.

16. Del Villar, I., et al., Optimization of sensitivity in Long Period Fiber Gratings with overlay deposition. Opt. Express, 2005. 13(1): p. 56-69.

17. Cusano, A., et al., Mode transition in high refractive index coated long period gratings. Opt. Express, 2006. 14(1): p. 19-34.

18. Yang, J., et al., Long-period grating refractive index sensor with a modified cladding structure for large operational range and high sensitivity. Appl. Opt., 2006. 45(24): p. 6142-6147.

19. Chung, K.-W. and S. Yin, Analysis of a widely tunable long-period grating by use of an ultrathincladding layer and higher-ordercladding mode coupling. Opt. Lett., 2004. 29(8): p. 812-814.

20. Chiavaioli, F., et al., Towards sensitive label-free immunosensing by means of turn-around point long period fiber gratings. Biosensors and Bioelectronics, 2014. 60(0): p. 305-310.

21. MacDougall, T.W., et al., Generalized expression for the growth of long period gratings. Photonics Technology Letters, IEEE, 1998. 10(10): p. 1449-1451.

22. X. Shu, Y.L., D. Zhao, B. Gwandu, F. Floreani, L. Zhang, and I. Bennion, Dependence of temperature and strain coefficients on fiber grating type and its application to simultaneous temperature and strain measurement. Opt. Lett., 2002. 27: p. 701-703.

23. Xuewen, S., et al., Room-temperature operation of widely tunable loss filter. Electronics Letters, 2001. 37(4): p. 216-218.

24. Yan, Z., et al., Broadband Tunable All-Fiber Polarization Interference Filter Based on $45^{\circ}$; Tilted Fiber Gratings. J. Lightwave Technol., 2013. 31(1): p. 94-98. 